\title{
Efficient Cycle Frequency Acquisition of a Cyclostationary Signal with the FACA Method
}

\author{
Yi HU ${ }^{1,2}$, Baoguo $Y U^{1}$, Zhixin DENG ${ }^{1}$, Guodong $\mathrm{HE}^{3}$, Haijun $Z H O U^{2}$ \\ ${ }^{1}$ State Key Laboratory of Satellite Navigation System \& Equipment Technology \\ ${ }^{2}$ School of Electronics \& Electrical Engineering, Chuzhou University, Chuzhou, China \\ ${ }^{3}$ School of Physics \& Electronic Information, Anhui Normal University, Wuhu, China \\ hygps607@163.com,yubg@sina.cn, cetc54007@ta.an, \{gdhe, hjzhou\}@163.com
}

Submitted December 31, 2018 / Accepted April 14, 2019

\begin{abstract}
The cycle frequencies of a cyclostationary signal can be used for the signal identification and classification, separation of the overlapped signals in cycle domain, and so on. Efficient cycle frequency acquisition depends on the fast measurement of cyclic autocorrelation function (CAF) or spectral correlation function (SCF) of the signal. Presently the relative efficient $C A F$ and SCF measuring methods mainly include the cyclic correlogram, the well-known fast Fourier transform accumulation method (FAM), and so on. Motivated by these methods, a new efficient cycle frequency acquisition method which integrates the fast Fourier transform (FFT) algorithm with the autocorrelated cyclic autocorrelation function, named FACA, is presented. With the presented method, we can acquire the cycle frequencies of a cyclostationary signal more efficiently with a given level of reliability. Meanwhile, by enlarging the FFT window width of the FACA method we can get the same cycle frequency resolution as the benchmarked method FAM, but the computation cost still can be spared at this case.
\end{abstract}

\section{Keywords}

Cycle frequency acquisition, cyclostationary signal, FACA method, FFT algorithm.

\section{Introduction}

For many man-made signals, they take the features of cyclostationarity which arise from their periodic sampling, timing, carrier and/or data symbol/bit modulation, and so on [1], [2]. At the same time, different signals often take different features of cyclostationarity due to their different periodic parameters, or more precisely, their different cycle frequencies [2]. On the other hand, the cycle frequencies of a cyclostationary signal can be used to detect the presence of the signal, or to discriminate the signal from noise or other interfering signal for the purpose of signal identification and classification as used in cognitive radio [3-5] or cognitive positioning system [6], [7], and so on. Moreover, in the applications of frequency-shift (FRESH) filtering for spatial-time or time-frequency overlapped signals [8-10], the difference in cycle frequencies of different signals can also be used to separate the overlapped signal of interest (SOI) from other signals.

To acquire the cycle frequencies of a cyclostationary signal efficiently, first and critical step is the fast measurement of CAF or SCF of the signal $[1,2,11,12]$. Presently the relative efficient measuring methods of CAF and SCF mainly include autocorrelated cyclic auto-correlation (ACA) [13], cyclic correlogram [1], the well-known fast Fourier transform accumulation method (FAM) [12], [14], strip spectral correlation algorithm (SSCA) [12], fast spectral correlation algorithm (Fast-SC) [15], and so on. Generally speaking, the SCF-based methods often outperform the CAF-based ones in cycle frequency resolution due to their constraints on reliability of the acquired cycle frequencies $[1,12,14]$, but this does not exist in CAF-based methods [2], [11]. At the same time, finer cycle frequency resolution means that more computation and storage will cost in measuring the SCF of the received signal, and this will accordingly render the SCF-based methods are inferior to the CAF-based ones in cycle frequency acquisition efficiency. For example, to acquire the cycle frequencies of the weak signal embedded in noise with the SCF-based method, often more collected data is required so that the finer cycle frequency resolution can be met, and this will accordingly increase the computation cost; while for the CAF-based methods, since there are no constraints on their cycle frequency resolutions, they can acquire the cycle frequencies more quickly with relatively shorter collected data [13]. Reasonably, the shorter collected data means that the CAFbased methods are often inferior to the SCF-based ones in cycle frequency resolution.

The further analysis on efficient SCF measuring methods such as FAM and Fast-SC shows that the critical step adopted in these methods is the use of fast Fourier transform (FFT) algorithm to speed up the measuring process [12], [15]. While the analysis on efficient CAF measuring methods such as cyclic correlogram and ACA shows 
that they can improve the measuring efficiency by using a relative smaller data-tapering window to decrease the cycle frequency resolution [1], [13]. Motivated by these methods, a new efficient cycle frequency acquiring method named FACA which integrates the FFT algorithm and the time-average operation with the ACA function is presented. By this new method, we can acquire the cycle frequencies of a cyclostationary signal more quickly with a given level of reliability, meanwhile, if we enlarge the FFT window width of the FACA method, we can get the same cycle frequency resolution as the benchmarked method FAM, but the computation cost still can be spared at this case.

The rest of the paper is organized as follows. In Sec. 2, CAF and SCF of the received signal and their measurements are briefly reviewed. Section 3 presents the detailed derivations of the presented FACA method, along with its implementation and computation cost. In Sec. 4, the performance of the given FACA method is simulated compared with other methods. Finally, the paper is concluded in Sec. 5.

\section{Cyclostationarity of the Signal}

\subsection{CAF and SCF}

For the received signal $x(t)$, it can be formulated as

$$
x(t)=s(t)+w(t)
$$

where $s(t)$ is the SOI, $w(t)$ is the interfering signal including the noise. For simplicity, we assume that $w(t)$ is a weak stationary signal and not cyclic correlated with $s(t)$ in the following analysis, otherwise if $w(t)$ is also cyclostationary and stronger than $s(t)$, we can use the presented FACA method to get the cycle frequencies of $w(t)$ first, and then cancel it from $x(t)$ with the FRESH filtering [16], [17] to get the signal of $s(t)$.

Based on (1), CAF and the corresponding SCF of $x(t)$ can be respectively given as [8]

$$
\begin{gathered}
R_{x}^{\alpha}(\tau)=\left\langle x(t+\tau / 2) x^{*}(t-\tau / 2) \exp (-\mathrm{j} 2 \pi \alpha t)\right\rangle, \\
S_{x}^{\alpha}(f)=\int_{-\infty}^{\infty} R_{x}^{\alpha}(\tau) \exp (-\mathrm{j} 2 \pi f \tau) \mathrm{d} \tau
\end{gathered}
$$

where $\langle\cdot\rangle$ denotes the time-average, the superscript "*" denotes the complex conjugate, $\tau$ is the time delay, $\alpha$ is the cycle frequency of $x(t)$ and $\alpha \neq 0$.

$$
\begin{aligned}
& \text { Substitute (2) into (3) and it will yield } \\
& \qquad S_{x}^{\alpha}(f)=\lim _{T \rightarrow \infty} T\left\langle X_{T}(t, f+\alpha / 2) X_{T}^{*}(t, f-\alpha / 2)\right\rangle
\end{aligned}
$$

where

$$
X_{T}(t, f)=\frac{1}{T} \int_{t-T / 2}^{t+T / 2} x(u) \exp (-\mathrm{j} 2 \pi f u) \mathrm{d} u
$$

For conjugate CAF of $x(t)$ and its SCF, they have the similar relationships as (2) to (5). In addition, the discrete time CAF and the corresponding SCF of $x(t)$ are respectively defined as [1]

$$
\begin{array}{r}
R_{x}^{\alpha}(\tau) \triangleq \lim _{L \rightarrow \infty} \frac{1}{2 L+1} \sum_{k=-L}^{L} x^{*}(k) x(k-\tau) \exp \left[-\mathrm{j} 2 \pi \alpha(k-\tau / 2) T_{\mathrm{s}}\right] \\
S_{x}^{\alpha}(f) \triangleq \sum_{\tau=-\infty}^{+\infty} R_{x}^{\alpha}(\tau) \exp \left(-\mathrm{j} 2 \pi f \tau T_{\mathrm{s}}\right)
\end{array}
$$

where $T_{\mathrm{S}}=1 / f_{\mathrm{S}}$ is the time sampling interval, $f_{\mathrm{S}}$ is the sampling frequency, and $x(k) \triangleq x\left(k T_{\mathrm{s}}\right)$.

\subsection{Measurements of CAF and SCF}

For $x(k)$ with finite collected data, its CAF can be measured by time-averaged cyclic correlogram (TCC) [2]

$$
\tilde{R}_{x}^{\alpha}(\tau)_{\Delta t}=\frac{1}{L N} \sum_{k=0}^{L-1} \sum_{v=k}^{k+N-1} x^{*}(v) x(v-\tau) \exp \left[-\mathrm{j} 2 \pi \alpha(v-\tau / 2) T_{\mathrm{s}}\right]
$$

where $L=\left\lfloor f_{\mathrm{s}} \Delta t\right\rfloor$ is the number of samples in time span $\Delta t$ and $\lfloor\cdot\rfloor$ denotes the floor operation.

While for SCF of $x(k)$ with finite collected data, it can be measured by time or frequency-averaged cyclic periodogram [1], [14], in which the time-averaged cyclic periodogram can be given as

$$
\tilde{S}_{X_{N}}^{\alpha}(f)_{\Delta t}=\frac{N}{L} \sum_{k=0}^{L-1} \sum_{v=0}^{N-1} X_{N}^{*}(k-v, f-\alpha / 2) X_{N}(k-v, f+\alpha / 2)
$$

where

$$
X_{N}(v, f)=\frac{1}{N} \sum_{m=-N / 2}^{N / 2-1} g_{N}(m) x(v-m) \exp \left[-\mathrm{j} 2 \pi f(v-m) T_{\mathrm{s}}\right]
$$

where $g_{N}(\bullet)$ is a $N$-point data-tapering window.

To get the reliable measurement of SCF, in (9) the cycle frequency resolution of $\tilde{S}_{X_{N}}^{\alpha}(f)_{\Delta t}$ denoted by $\Delta \alpha$ and the frequency resolution denoted by $\Delta f$ should meet the reliability condition [1], [12]

$$
\Delta f / \Delta \alpha=\Delta f \Delta t \approx L / N \gg 1 \text {. }
$$

\section{Cycle Frequency Acquisition with the FACA Method}

From (9) and (11), we can see that the computation cost of SCF is high, especially when the number of collected data or oversampling factor is large. To this problem, we can make a tradeoff between the computation cost and the cycle frequency resolution with a given level of reliability, and this is just the motivation of the proposed FACA method. 


\subsection{Derivation of the FACA Method}

From (8) the segmented CAF of $x(k)$ can be given as

$$
\tilde{R}_{x}^{\alpha}(k, \tau)_{N}=\frac{1}{N} \sum_{v=k}^{k+N-1} x^{*}(v) x(v-\tau) \exp \left[-\mathrm{j} 2 \pi \alpha(v-\tau / 2) T_{\mathrm{s}}\right]
$$

where $N$ is the length of each segmented data block, and time delay $\tau$ meets $|\tau| \leq N-1$.

Based on (12) and the definition of ACA given in [13], the ACA of $\tilde{R}_{x}^{\alpha}(k, \tau)_{N}$ can be written as

$$
R_{R}(\tau, \alpha ; D)_{N}=\frac{1}{Z} \sum_{k=0}^{Z-1} \tilde{R}_{x}^{\alpha}(k, \tau)_{N}^{*} \tilde{R}_{x}^{\alpha}(k-D, \tau)_{N}
$$

where $D$ is a lag parameter of ACA whose optimal value equals $N$ [13] , and $Z$ is the number of total collected data.

In (13) if let $\alpha \triangleq \alpha_{l} f_{\mathrm{s}} / L$ where $N \ll L \leq Z, \alpha_{l}$ is a non-negative integer and $0 \leq \alpha_{l} \leq L-1$, then (13) will be changed to

$$
R_{R}\left(\tau, \alpha_{l} ; D\right)_{N}=\frac{1}{Z} \sum_{k=0}^{Z-1} \tilde{R}_{x}^{\alpha_{l}}(k, \tau)_{N}^{*} \tilde{R}_{x}^{\alpha_{l}}(k-D, \tau)_{N}
$$

Due that $Z \gg N$, the calculation of $R_{R}\left(\tau, \alpha_{l} ; D\right)_{N}$ in (14) is time consuming, practically it can be fulfilled by the segmented time-average method as

$$
\begin{aligned}
& R_{R}\left(\tau, \alpha_{l} ; D\right)_{N}=\frac{1}{P} \sum_{i=0}^{P-1} \tilde{R}_{x}^{\alpha_{l}}(i K, \tau)_{N}^{*} \tilde{R}_{x}^{\alpha_{l}}(i K-D, \tau)_{N}
\end{aligned}
$$

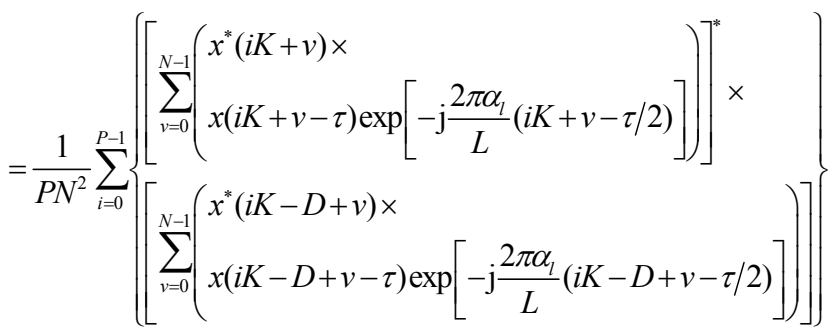

where $K$ is the offset of two adjacent segmented data blocks, $P=\left\lfloor\left[Z-\left(2 \tau_{\max }+1\right)-N-D+K\right] / K\right\rfloor$ is the $\max$ block number, and $\tau_{\max }$ is the max time delay where $\tau_{\max }>0$.

In (14) the condition $L \gg N$ means that the computation of $R_{R}\left(\tau, \alpha_{l} ; D\right)_{N}$ for $0 \leq \alpha_{l} \leq L-1$ is still very high. As a trade-off, we can improve its efficiency by decreasing the cycle frequency resolution of $x(k)$ from $f_{\mathrm{S}} / L$ to $f_{\mathrm{S}} / N$ where $N$ determines the level of reliability, meanwhile, if let $r_{x}(v ; i K, \tau) \triangleq x^{*}(i K+v) x(i K+v-\tau) \quad$ in (15), where $v=0,1, \cdots, N-1$, we will find that $\tilde{R}_{x}^{\alpha_{l}}(i K, \tau)_{N}$ can be implemented with FFT algorithm, i.e.,

$$
\begin{aligned}
\tilde{R}_{x}^{\alpha_{l}}(i K, \tau)_{N} & =\exp \left[-\mathrm{j} \frac{\pi \alpha_{l}(2 i K-\tau)}{N}\right] \underbrace{\sum_{v=0}^{N-1} r_{x}(v ; i K, \tau) \exp \left[-\mathrm{j} \frac{2 \pi v \alpha_{l}}{N}\right]}_{\text {FFT }} \\
& \triangleq \exp \left[-\mathrm{j} \frac{\pi \alpha_{l}(2 i K-\tau)}{N}\right] \mathcal{F}_{x}\left(\alpha_{l} ; i K, \tau\right)_{N}
\end{aligned}
$$

where $0 \leq \alpha_{l} \leq N-1$. In a similar way we also have

$$
\begin{aligned}
& \tilde{R}_{x}^{\alpha_{l}}(i K-D, \tau)_{N} \\
= & \exp \left[-\mathrm{j} \frac{\pi \alpha_{l}(2 i K-2 D-\tau)}{N}\right] \underbrace{\sum_{v=0}^{N-1} r_{x}(v ; i K-D, \tau) \exp \left[-\mathrm{j} \frac{2 \pi v \alpha_{l}}{N}\right]}_{\text {FFT }} \\
\triangleq & \exp \left[-\mathrm{j} \frac{\pi \alpha_{l}(2 i K-2 D-\tau)}{N}\right] \mathcal{F}_{x}\left(\alpha_{l} ; i K-D, \tau\right)_{N}
\end{aligned}
$$

where $r_{x}(v ; i K-D, \tau) \triangleq x^{*}(i K-D+v) x(i K-D+v-\tau)$ for $v=0,1, \cdots, N-1$.

Substitute (16) and (17) into (15), and take $D=N$, we can get the proposed FACA method as

$$
R_{R}\left(\tau, \alpha_{l}\right)_{N}=\frac{1}{P N^{2}} \sum_{i=0}^{P-1}\left(\begin{array}{l}
\mathcal{F}_{x}\left(\alpha_{l} ; i K, \tau\right)_{N} \times \\
\mathcal{F}_{x}^{*}\left(\alpha_{l} ; i K-N, \tau\right)_{N}
\end{array}\right) .
$$

If we rewrite back $\alpha_{l}$ to $\alpha$ for a general purpose, then we will have

$$
R_{R}(\tau, \alpha)_{N}=\frac{1}{P N^{2}} \sum_{i=0}^{P-1}\left(\begin{array}{l}
\mathcal{F}_{x}(\alpha ; i K, \tau)_{N} \times \\
\mathcal{F}_{x}^{*}(\alpha ; i K-N, \tau)_{N}
\end{array}\right)
$$

\subsection{Proof of the FACA Method}

Utilizing the assumption that $s(k)$ is not cyclic correlated with $w(k)$ for $\alpha \neq 0$, we can get the approximate result of $R_{R}(\tau, \alpha ; D)_{N}$ in (15) as [13]

$$
\begin{aligned}
R_{R}(\tau, \alpha ; D)_{N} \cong & \sum_{\alpha_{s} \in \mathcal{A}}\left|R_{s}^{\alpha_{s}}(\tau) G_{N}\left(\alpha_{\mathrm{s}}-\alpha\right)\right|^{2} \exp \left[\mathrm{j} 2 \pi\left(\alpha-\alpha_{\mathrm{s}}\right) D\right]+ \\
& \frac{1}{P} \sum_{i=0}^{P-1} W_{x}^{\alpha}(i K, \tau)_{N}^{*} W_{x}^{\alpha}(i K-D, \tau)_{N}
\end{aligned}
$$

where $\alpha_{s} \neq 0$ is a discrete cycle frequency of $s(k), \mathcal{A}_{s}$ is the cycle frequency set of $s(k)$, and

$$
G_{N}(\alpha)=\sin (N \pi \alpha) /(N \sin \pi \alpha)
$$

$$
W_{x}^{\alpha}(k, \tau)_{N}=\frac{1}{N} \sum_{v=k}^{k+N-1} w^{*}(v) w(v-\tau) \exp [-\mathrm{j} 2 \pi \alpha(v-\tau / 2)]
$$

Considering that $w(k)$ is stationary and using the result $R_{s}^{\alpha_{s}}(\tau)=R_{x}^{\alpha_{s}}(\tau)$ when $N$ approaches infinity for $\alpha_{s} \neq 0$ [18], we can approximately simplify (20) as 
$R_{R}(\tau, \alpha)_{N} \underset{N \gg 1}{\cong} \sum_{\substack{\alpha_{s} \in \mathcal{A} \\ \alpha_{s}}}\left|R_{x}^{\alpha_{s}}(\tau)\right|^{2} \delta\left(\alpha_{s}-\alpha\right) \exp \left[\mathrm{j} 2 \pi\left(\alpha-\alpha_{s}\right) N\right]$

$$
\alpha \neq 0 \text {. }
$$

From (23) we can get that $R_{R}(\tau, \alpha)_{N}$ will have a peak at $\alpha=\alpha_{s}$ where $\alpha \neq 0$, and the peak value equals $\left|R_{x}^{\alpha_{s}}(\tau)\right|^{2}$. On the other hand, equation (23) is equivalent to (19) for the same $R_{R}(\tau, \alpha)_{N}$ and this indicates we can efficiently acquire the cycle frequencies of $s(k)$ according to (19).

\subsection{Implementation of the FACA Method}

The analysis given above shows that we can acquire the cycle frequency $\alpha_{s}$ efficiently by searching the peak of $\left|R_{R}(\tau, \alpha)_{N}\right|$ in $(\tau, \alpha)$ plane excluding $\alpha=0$. With the derivation given in subsection 3.1, we can get the general implementation process of the presented FACA method as Fig. 1.

In Fig. 1, the accumulation of $\left|R_{R}(\tau, \alpha)_{N}\right|$ from $-\tau_{0}$ to $\tau_{0}$ is to strengthen the cycle frequency lines of $s(k)$, i.e.,

$$
R_{R}(\alpha)_{N}=\frac{1}{P N^{2}} \sum_{\tau=-\tau_{0}}^{\tau_{0}}\left|\sum_{i=0}^{P-1} \mathcal{F}_{x}^{*}(\alpha, i K ; \tau)_{N} \mathcal{F}_{x}(\alpha, i K-N ; \tau)_{N}\right|
$$

where $0<\tau_{0} \leq \tau_{\max }$.

It can be shown that in (23) and (24) the acquired cycle frequency $\hat{\alpha}_{s}=\arg \max _{\alpha(\neq 0)} R_{R}(\alpha)_{N}$ satisfies the condition that $\lim _{N \rightarrow \infty}(2 N+1)\left(\hat{\alpha}_{s}-\alpha_{s}\right)=0$ [19], and this means that $\hat{\alpha}_{s}$ is an asymptotic estimation of $\alpha_{s}$.
Additionally, when we apply the proposed FACA method to a given task, some critical parameters of the FACA method in Fig. 1 can be set as: $N$ is determined by the required cycle frequency resolution and it can take the value of power 2, for example, 4096 or 8192 and so on; $D$ can take the optimal value $N ; K$ can take any non-zero value and it is often chosen as $K=N ; P$ is defined in (15); $\tau_{\max }$ can take tens of time samples and $\tau_{\max } / 2 \leq \tau_{0} \leq \tau_{\max }$.

\subsection{Computational Cost of the FACA Method}

To find the decrease in computation cost of the proposed FACA method, the computational complexity of the FACA method compared with other three typical cycle frequency acquisition methods, i.e., TCC [2], FAM [12], and Fast-SC [15], is analyzed. For simplicity, we assume that $x(n)$ is a real-valued signal, meanwhile, since that the multiplication operation often takes more time than other operation in data processing, in the following we mainly focus on the comparisons of multiplication computations of four given methods.

From (16) we can derive that the complexity of $r_{x}(v ; i K, \tau)$ is $N$ when $v$ varies from $i K$ to $i K+N-1$, and $\mathcal{F}_{x}(\alpha ; i K, \tau)_{N}$ is $(N / 2) \log _{2} N$ for $0 \leq \alpha \leq N-1$; whereas the complexity of (17) is same as that of (16). With these results and notice that $R_{R}(-\tau, \alpha)_{N}=R_{R}(\tau, \alpha)_{N}$, we can get the computational complexity of $R_{R}(\tau, \alpha)_{N}$ in (19) for $|\tau| \leq \tau_{\max }$ is about

$$
\mathbb{C}_{\mathrm{FACA}} \sim\left(\tau_{\max }+1\right) P\left(N \log _{2} N+2 N\right)
$$

where $P=\left\lfloor\left[L-\left(2 \tau_{\max }+1\right)-2 N+K\right] / K\right\rfloor$ and $\tau_{\max } \ll N$.

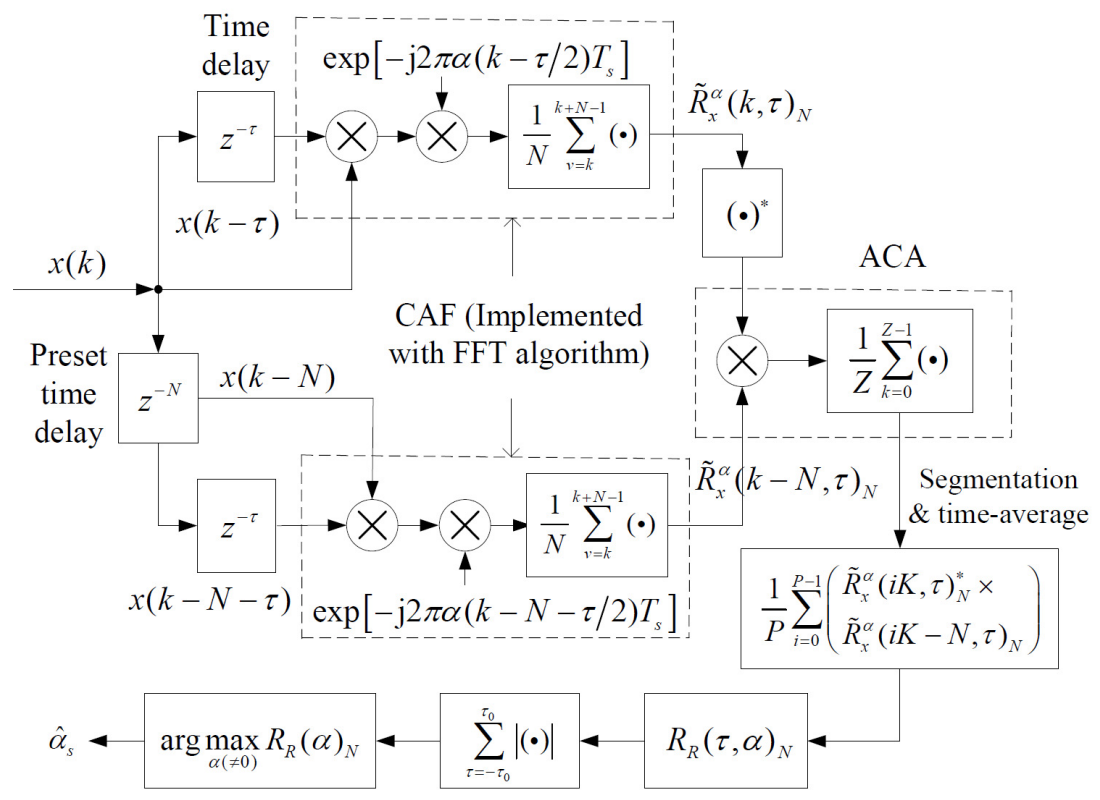

Fig. 1. Implementation process of the FACA method. 
For TCC, after dividing the collected data into $P$ blocks, the TCC of $\tilde{R}_{x}^{\alpha}(\tau)_{\Delta t}$ in (8) can be calculated by

$$
\tilde{R}_{x}^{\alpha}(\tau)_{\Delta t}=\frac{1}{P N} \sum_{i=0}^{P-1} \sum_{v=0}^{N-1}\left(\begin{array}{l}
x^{*}(i K+v) \times \\
x(i K+v-\tau) \exp \left[-\mathrm{j} 2 \pi \alpha(i K+v-\tau / 2) T_{\mathrm{s}}\right]
\end{array}\right) .
$$

Similarly, if we reduce the cycle frequency resolution of TCC from $f_{s} / L$ to $f_{s} / N$, then (26) can also be implemented with FFT algorithm as

$$
\begin{aligned}
& \tilde{R}_{x}^{\alpha}(\tau)_{\Delta t}= \\
& \frac{1}{P N} \sum_{i=0}^{P-1}[\exp \left[-\mathrm{j} 2 \pi \alpha(i K-\tau / 2) T_{\mathrm{s}}\right] \underbrace{\left.\sum_{v=0}^{N-1}\left(\begin{array}{l}
x^{*}(i K+v) \times \\
x(i K+v-\tau) \exp \left[-\mathrm{j} 2 \pi \alpha v T_{\mathrm{s}}\right.
\end{array}\right]\right)}_{\text {FFT }}] .
\end{aligned}
$$

Processing as the FACA method, we can give the computational complexity of TCC as

$$
\mathbb{C}_{\mathrm{TCC}} \sim\left(\tau_{\max }+1\right) P\left(\left(N \log _{2} N\right) / 2+2 N\right) .
$$

For FAM, its computational complexity is about [12], $[15]$

$$
\mathbb{C}_{\mathrm{FAM}} \sim \frac{Z N}{2 K}\left\{4+\log _{2} N+\frac{N}{2}\left[1+\log _{2}\left(\frac{Z}{K}\right)\right]\right\} .
$$
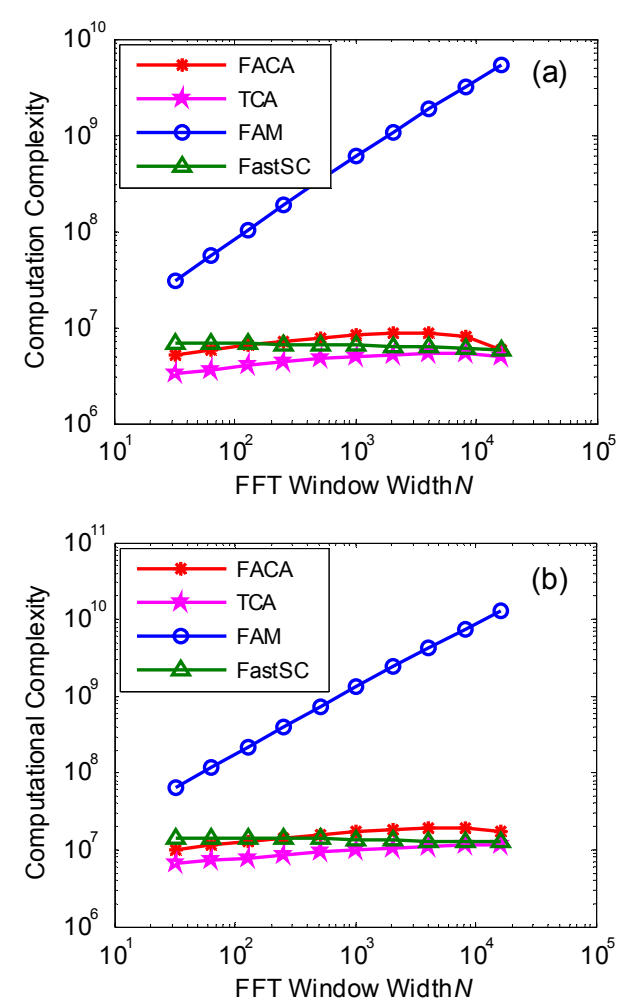

Fig. 2. Comparisons on computational complexity of the presented FACA method, TCC, FAM, and Fast-SC under different FFT window width and different data length $Z$, in which (a) $Z=65536$ and (b) $Z=131072$, and time delay $\tau_{\max }$ has been set to 10 time samples.
If we take the optimal value $K=N / 4$ [12], the result of (29) will become

$$
\mathbb{C}_{\mathrm{FAM}}^{\mathrm{opt}} \sim 3 N Z+2 Z \log _{2} N+Z N \log _{2}(Z / N)+8 Z .
$$

While for the Fast-SC method, based on the result given in [15], its computational complexity can be read as

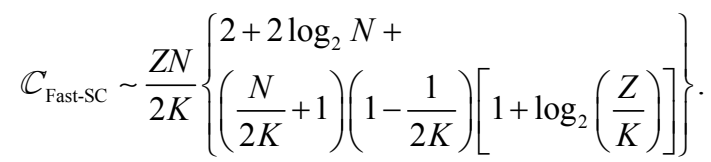

To view roughly the results given above, simulations on computational complexity of FACA, TCC, FAM, and Fast-SC under different data length are shown as Fig. 2, in which the chosen FFT window width $N=\{32,64,128$, $512,1024,2048,4096,8192,16384\}$. Note that in Fig. 2, we have used $K=N$ for FACA and TCC methods; while for FAM and Fast-SC methods, $K$ should not be larger than $N / 4$ so as to reduce the cycle leakage [1], and we have chosen $K=N / 4$.

From Fig. 2, we can see that the computational complexity of the presented FACA method is lower than FAM, a little higher than TCC, and is close to Fast-SC. But at the same time, it will be found in the follows that from Fast-SC we can hardly get the non-conjugate cycle frequencies [20] related to the carrier frequency except the conjugate cycle frequency related to the data symbol or bit rate. For TCC, though its complexity is lowest, its cycle frequency acquisition performance is poorer than the presented FACA method, and this will also be seen in following simulations.

\section{Performance Verifications}

To further verify the cycle frequency acquisition performance of the presented FACA method, an example that a binary phase-shift keying (BPSK) signal corrupted by the additive Gaussian noise is studied. In detail the scenarios that (i) cycle frequency acquisition results, (ii) cycle frequency acquisition error, and (iii) cycle frequency acquisition probability of the BPSK signal are simulated.

The common parameters used in the simulations are set as:

- Sampling frequency $f_{\mathrm{s}}=20 \mathrm{MHz}$, and data collected time is 1.5 milliseconds;

- For FACA and TCC, their max time delay and time lag accumulation value are set to $\tau_{\max }=15$ time samples and $\tau_{0}=10$ time samples, respectively;

- Two fundamental cycle frequencies of the BPSK signal [8], [20] are set as: carrier frequency $2 f_{\mathrm{c}}=$ $4.109 \mathrm{MHz}$, and data bit rate $R_{\mathrm{b}}=1.2288 \mathrm{Mbit} / \mathrm{s}$;

- The chosen FFT window width $N=\{32,64,128,512$, 1024, 2048, 4096, 8192\}; 
- $\Delta \alpha$ and $\Delta f$ of the benchmarked FAM method are fixed to $1 \mathrm{kHz}$ and $260 \mathrm{kHz}$ respectively to meet the reliability condition of (11).

Other uncommon parameters will be given in detailed simulations.

\subsection{Acquisition of the Cycle Frequencies}

In this subsection, the surface plots of the BPSK signal obtained with the FACA method, TCC, FAM and Fast$\mathrm{SC}$, and their profiles related to the cycle frequencies of $2 f_{\mathrm{c}}$ and $R_{\mathrm{b}}$ are simulated. The results are shown as Figs. 3 and 4, in which Figs. 4(b), 4(d), 4(e) and 4(f) are corresponding to the profiles of Figs. 3(a) to 3(d), and the FFT window width of the Fast-SC method used in Figs. 3(d) and 4(f) is set to $N=128$.

From Figs. 4(a) to 4(d) we can see that the cycle frequency acquisition results of the FACA method are less affected by the FFT window width than those of the TCC. For example, when $N=1024$, we can hardly get the cycle frequencies of $2 f_{\mathrm{c}}$ and $R_{\mathrm{b}}$ from the "noisy" cycle frequency lines of TCC, and only when $N=8192$ can we get the relative better cycle frequency lines, and hence the better results of $2 f_{\mathrm{c}}$ and $R_{\mathrm{b}}$; but from the presented FACA method we can all well acquire the cycle frequencies of $2 f_{\mathrm{c}}$ and $R_{\mathrm{b}}$ at the two cases. In addition, the result of Fig. 4(f) also shows that from Fast-SC we can hardly get the nonconjugate cycle frequencies related to $2 f_{\mathrm{c}}$ except the conjugate cycle frequencies related to the data bit rate $R_{\mathrm{b}}$.
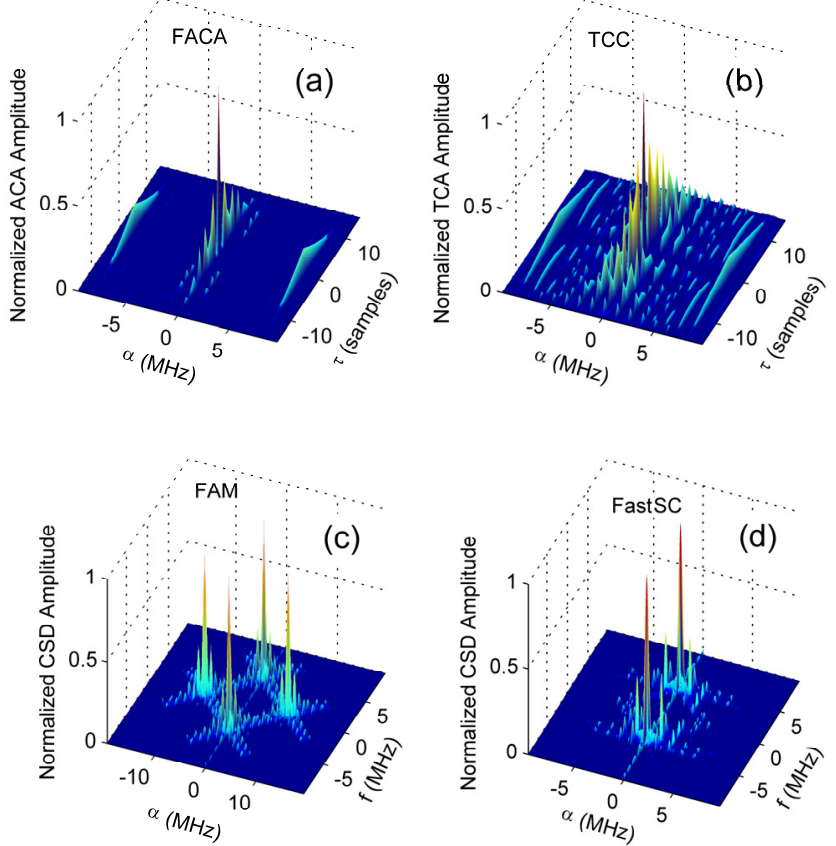

Fig. 3. Surface plots of the received signal obtained with: (a) FACA, (b) TCC, (c) FAM, and (d) Fast-SC. The FFT window width used in FACA and TCC is 8192 , and the input SNR of the BPSK signal equals $5 \mathrm{~dB}$.
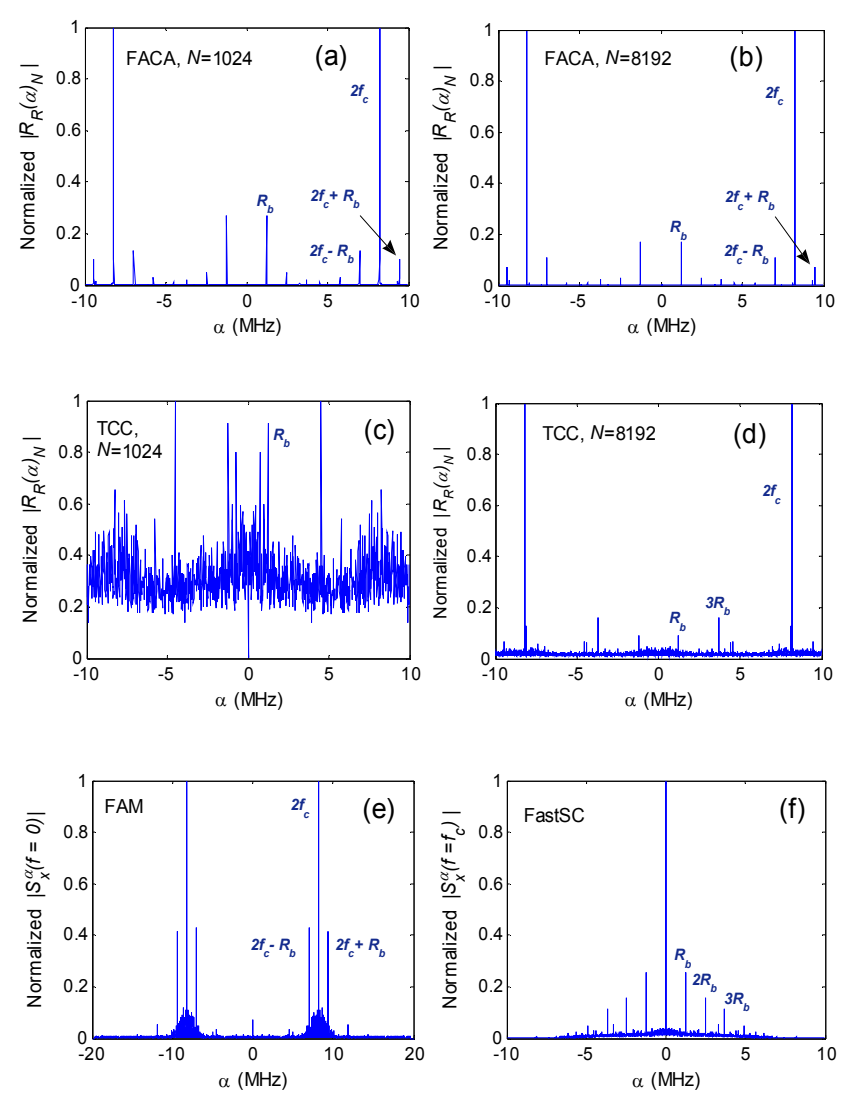

Fig. 4. Profiles of the FACA method, TCC, FAM and Fast-SC based on Fig. 3. Here in Figs. 4(a) to 4(d), the DC components of FACA and TCC methods at $\alpha=0$ have been cancelled.

Also the results of Figs. 3 and 4 can be explained by that for the same input signal to noise ratio (SNR) of the BPSK signal, the output SNRs after processing with four given methods are different. In general case, the analytical results of the output SNRs of four given methods are hard to get, but if we regard the methods of FACA and TCC as the fast implementations of ACA and the time-variant finite-average cyclic autocorrelation (CA), respectively, and the methods of FAM and Fast-SC as two different fast implementations of the temporally smoothed cyclic periodogram (SCP), we can use the results given in [13] and [15] for a rough perception of the output SNRs of four given methods and which can be rewritten as: the FACA is about $(P K+2 N) N B^{2} \rho_{A}^{4} /\left(2 f_{\mathrm{s}}^{2} \xi_{B}^{2}(\alpha)\right)$, the TCC is about $(P K+N) B \rho_{A}^{2} /\left(K_{A} f_{s} \xi_{B}(\alpha)\right)$, the FAM is about $1 /\left(K_{P} N\right) \times$ $(P K+N) \rho_{P}^{2}$ and the result is also approximately fit for the Fast-SC, where $B$ is the noise bandwidth, $\xi_{\mathrm{B}}=1-|\alpha| / B$ if $|\alpha| \leq B$ and 0 otherwise, and the definitions of $\rho_{A}, \rho_{P}, K_{A}$, and $K_{p}$ can be referred to [13]. From these results, we can easily get that for the CAF-based methods of FACA and TCC, the output SNR of the former is much better than that of the latter when the input SNR $\rho_{A} \geq 1$, as is also clearly shown in Figs. 3(a) and 3(b) and Figs. 4(a) to 4(d). 


\subsection{RMSE of the Acquired Cycle Frequencies}

To check the cycle frequency acquisition performance under different input SNR, the relative root mean square error (RMSE) of $2 f_{\mathrm{c}}$ and $R_{\mathrm{b}}$ of the BPSK signal acquired by FACA, TCC, FAM, and Fast-SC are simulated, and the results are shown as Fig. 5.

From Fig. 5 we can see that both the relative RMSEs of $2 f_{\mathrm{c}}$ and $R_{\mathrm{b}}$ acquired by the FACA method decrease with the increase of FFT window width $N$, and when $N$ equals 8192 the results will become same or almost the same as the benchmarked method FAM. While for TCC, it shows in Fig. 5 that both the cycle frequencies of $2 f_{\mathrm{c}}$ and $R_{\mathrm{b}}$ cannot be acquired when $N$ is less than 1024, and only when $N$ is equal or larger than 4096 can we get the relative better cycle frequency acquisition results.

Figure 5 also shows that the performance of FAM is the best of all due to the constraint of reliability condition, and its cycle frequency resolution has been preset to $1 \mathrm{kHz}$, which leads to the unchanged RMSEs of $2 f_{\mathrm{c}}$ and $R_{\mathrm{b}}$ in Fig. 5; besides, the results of FAM for $N$ larger than 256 are not simulated because their computation time becomes fairly long at these cases. While for Fast-SC, its performance on $R_{\mathrm{b}}$ is also superior to the FACA method due to the requirement of reliability condition, but its use of varied FFT window width in cycle frequency acquisition leads to the varied RMSE results, meanwhile, the disability of acquiring non-conjugate cycle frequencies related carrier
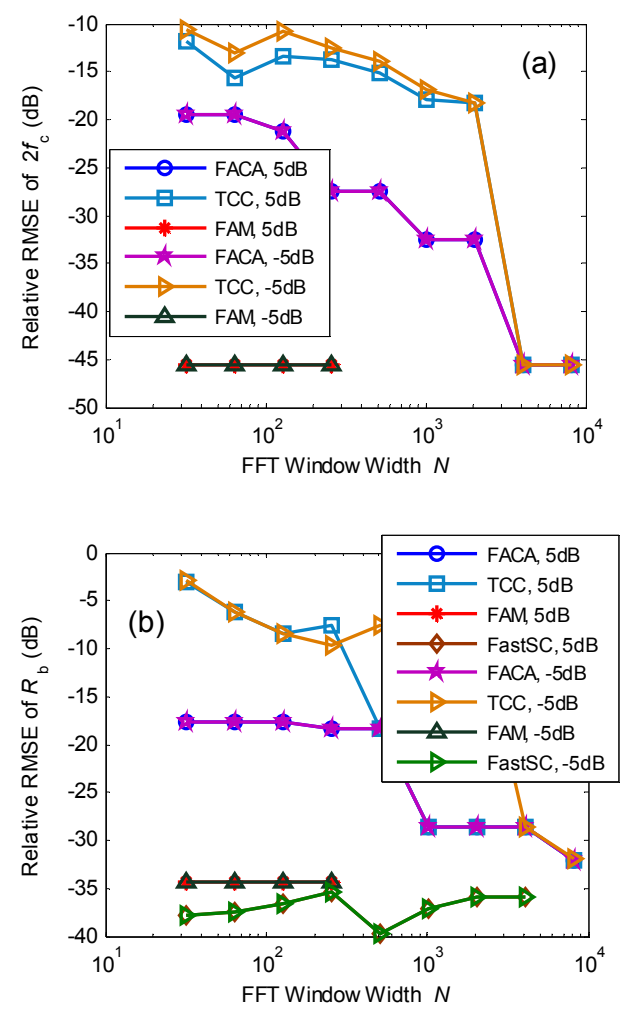

Fig. 5. Relative RMSEs of (a) $2 f_{\mathrm{c}}$ and (b) $R_{\mathrm{b}}$ acquired with the FACA method compared with the methods of TCC, FAM, and Fast-SC under different input SNR. frequency may limit its applications in some fields. General speaking, considering the cycle frequency acquisition performance and the computation complexity given before, the presented FACA is optimal among four given methods.

\subsection{Acquisition Probability of the Cycle Frequencies}

In this subsection, the effect of input SNR on acquisition probabilities of two fundamental cycle frequencies $2 f_{\mathrm{c}}$ and $R_{\mathrm{b}}$ with the FACA method are simulated by the Monte Carlo method, and the results which are the averages of 500 independent runs are shown as Fig. 6. In addition, in Fig.6 the results of TCC, FAM, and Fast-SC are also simulated for comparisons.

From Fig. 6(a) we can see that the acquisition probability of $2 f_{\mathrm{c}}$ obtained by the FACA method is almost the same as the results obtained by TCC and FAM. At the same time, for Fast-SC there is still no result shown in Fig. 6(a) due to its disability of obtaining the cycle frequencies related the carrier frequency.

Whereas in Fig. 6(b), we can see that with all methods of FACA, TCC, FAM, and Fast-SC, we can acquire the cycle frequency $R_{\mathrm{b}}$, but their acquisition probabilities are different. The simple comparisons show that the acquisition probability of the FACA method is the best, and the
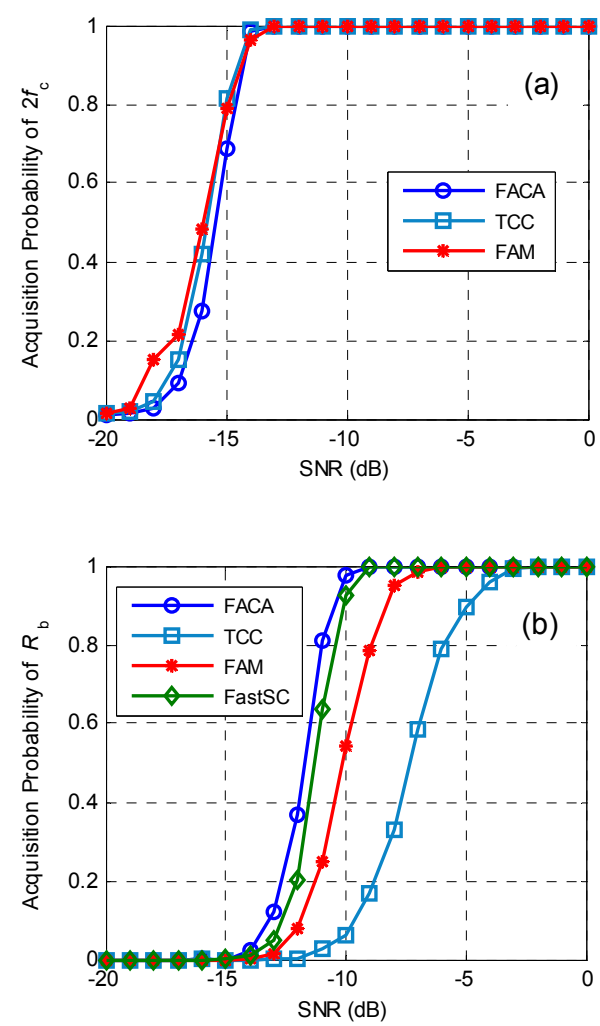

Fig. 6. Acquisition probabilities of (a) $2 f_{\mathrm{c}}$ and (b) $R_{\mathrm{b}}$ with the FACA method compared with the methods of TCC, FAM, and Fast-SC under different input SNR. The FFT window width used in FACA and TCC is 8192 . 
method of TCC is the poorest. This can be explained by that two rounds of correlations used in the FACA method can dramatically cancel the effect of noise, but for TCC there is only one round of correlation. For FAM and Fast$\mathrm{SC}$, their relative high computational cost can ensure better cycle frequency acquisition performance when input SNR is low. The results given in Fig. 6 are also consistent with the results given in Figs. 4 and 5.

\section{Conclusions}

An efficient cycle frequency acquisition method named FACA is presented. With the given method, we can acquire the cycle frequency or cycle frequencies of a cyclostationary signal more effectively with a given level of reliability. Meanwhile, by increasing the FFT window width of the given FACA method, we can get the same cycle frequency acquisition performance as the well-known FAM method. The final simulations on the acquisition results and acquisition performance of the BPSK signal validate the effectiveness of the presented method. In practice, the advantage of the presented FACA method makes it fit for the applications as fast spectrum detection in cognitive radio, interference mitigation in wireless communications or global positioning system and so on.

\section{Acknowledgments}

This work was supported by the Open Research Fund of State Key Laboratory of Satellite Navigation System \& Equipment Technology under grant CEPNT-2017KF-06, and the Natural Science Foundation of the Anhui Higher Education Institutions under grant KJ2018A0427. The authors would also like to acknowledge the anonymous reviewers for their constructive comments.

\section{References}

[1] GARDNER, W. A. Measurement of spectral correlation. IEEE Transactions on Acoustics, Speech, Signal Processing, 1986, vol. 34, no. 5, p. 1111-1123. DOI: $10.1109 /$ tassp.1986.1164951

[2] NAPOLITANO, A. Cyclostationarity: new trends and applications. Signal Processing, 2016, vol. 120, p. 385-408. DOI: 10.1016/j. sigpro.2015.09.011

[3] DOBRE, O. A. Signal identification for emerging intelligent radios: classical problems and new challenges. IEEE Instrumentation \& Measurement Magazine, 2015, vol. 18, no. 2, p. 11-18. DOI: $10.1109 / \mathrm{mim} .2015 .7066677$

[4] YAWADA, P. S., WEI, A. J. Cyclostationary detection based on non-cooperative spectrum sensing in cognitive radio network. In IEEE International Conference on Cyber Technology in Automation, Control, and Intelligent Systems. Chengdu (China), 2016, p. 184-187. DOI: 10.1109/cyber.2016.7574819

[5] DOBRE, O. A., ABDI, A., BAR-NESS, Y., et al. Cyclostationarity based blind classification of analog and digital modulations. In
Proceedings of the 2006 IEEE Conference on Military Communications. Washington (USA), 2006, p. 2176-2182. DOI: 10.1109/milcom.2006.302556

[6] SAHMOUDI, M., YANG, C., CALMETTES, V. The merits of the cyclostationarity of BOC signals for a cognitive GNSS receiver design. In IEEE/ION Position, Location and Navigation Symposium. Indian Wells (CA, USA), 2010, p. 1181-1188. DOI: 10.1109 /plans.2010.5507238

[7] LOHAN, E. S., LUNDÉN, J., SECO-GRANADOS, G., et al. Cyclic frequencies of BOC-modulated GNSS signals and their potential within a cognitive positioning framework. Navigation: Journal of the Institute of Navigation, 2014, vol. 61, no. 2, p. 95-114. DOI: $10.1002 /$ navi.59

[8] GARDNER, W. A. Cyclic Wiener filtering: theory and method. IEEE Transactions on Communications, 1993, vol. 41, no. 1, p. 151-163. DOI: $10.1109 / 26.212375$

[9] ZHANG, J., WONG, K. M., LUO, Z. Q., et al. Blind adaptive FRESH filtering for signal extraction. IEEE Transactions on Signal Processing, 1999, vol. 47, no. 5, p. 1397-1402. DOI: $10.1109 / 78.757230$

[10] CHOPRA, R., GHOSH, D., MEHRA, D. K. Spectrum sensing for cognitive radios based on space-time FRESH filtering. IEEE Transactions on Wireless Communications, 2014, vol. 13, no. 7, p. 3903-3913. DOI: $10.1109 /$ twc.2014.2314125

[11] ŠEBESTA, V., MARŠÁLEK, R., FEDRA, Z. OFDM signal detector based on cyclic autocorrelation function and its properties. $R a$ dioengineering, 2011, vol. 20, no. 4, p. 926-931. ISSN: 1210-2512

[12] ROBERTS, R. S., BROWN, W. A., LOOMIS, H. H. Computationally efficient algorithms for cyclic spectral analysis. IEEE Signal Processing Magazine, 1991, vol. 8, no. 2, p. 38-49. DOI: $10.1109 / 79.81008$

[13] YEUNG, G. K., GARDNER, W. A. Search-efficient methods of detection of cyclostationary signals. IEEE Transactions on Signal Processing, 1996, vol. 44, no. 5, p. 1214-1223. DOI: 10.1109/78.502333

[14] BROWN, W. A., LOOMIS, H. H. Digital implementations of spectral correlation analyzers. IEEE Transactions on Signal Processing, 1993, vol. 41, no. 2, p. 703-720. DOI: 10.1109/78.193211

[15] ANTONI, J., XIN, G., HAMZAOUI, N. Fast computation of the spectral correlation. Mechanical Systems and Signal Processing, 2017, vol. 92, p. 248-277. DOI: 10.1016/j.ymssp.2017.01.011

[16] HU, Y., SONG, M. Z., DANG, X. Y., et al. Interference mitigation for the GPS receiver utilizing the cyclic spectral analysis and RRMSWF algorithm. Radioengineering, 2017, vol. 26, no. 3, p. 798-807. DOI: $10.13164 /$ re.2017.0798

[17] YESTE-OJEDA, O. A., GRAJAL, J. Cyclostationarity-based signal separation in interceptors based on a single sensor. In IEEE Radar Conference. Rome (Italy), 2008, p. 1-6. DOI: 10.1109/radar.2008.4720750

[18] NAPOLITANO, A. Cyclostationarity: limits and generalizations. Signal Processing, 2016, vol. 120, p. 323-347. DOI: 10.1016/j.sigpro.2015.09.013

[19] CiBlat, P., LOUBATON, P., SERPEDIN, E., et al. Asymptotic analysis of blind cyclic correlation-based symbol-rate estimators. IEEE Transactions on Information Theory, 2002, vol. 48, no. 7, p. 1922-1934. DOI: 10.1109/tit.2002.1013133

[20] GELLI, G., PAURA, L., TULINO, A. M. Cyclostationarity-based filtering for narrowband interference suppression in directsequence spread-spectrum systems. IEEE Journal on Selected Areas in Communications, 1998, vol. 16, no. 9, p. 1747-1755. DOI: $10.1109 / 49.737643$ 


\section{About the Authors ...}

Yi HU was born in Lu'an, China, in 1974. He is currently an associate professor in the School of Electronics \& Electronic Engineering of Chuzhou University. He received his $\mathrm{PhD}$. degree from Nanjing University of Aeronautics \& Astronautics (NUAA) in 2015. His current research interests include satellite navigation signal processing and Sat-COM.

Baoguo YU was born in Inner Mongolia, China, in 1966. $\mathrm{He}$ is a research professorship in State Key Laboratory of Satellite Navigation System \& Equipment Technology. His current research interests include satellite navigation signal processing techniques and Sat-COM.
Zhixing DENG was born in Shijiazhuang, China, in 1982. $\mathrm{He}$ is a senior engineer in State Key Laboratory of Satellite Navigation System \& Equipment Technology. His current research interests include satellite navigation signal processing techniques and wireless communications.

Guodong HE was born in Anqing, China, in 1980. He is an associate professor in the School of Physics and Electronic Information of Anhui Normal University. His current research interests include signal processing techniques and wireless communications.

Haijun ZHOU was born in Wuhan, China, in 1986. He is a lecturer in the Department of Electrical Engineering of Chuzhou University. His current research interests include signal and electronic information processing techniques. 